\title{
Magnetization process in a frustrated plaquette dimerized ladder
}

\author{
F. Elias, M. Arlego, and C. A. Lamas \\ IFLP-CONICET, Departamento de Física, Facultad de Ciencias Exactas, \\ Universidad Nacional de La Plata, C.C. 67, 1900 La Plata, Argentina
}

(Received 10 April 2017; published 30 June 2017)

\begin{abstract}
The magnetic phase diagram of a plaquette dimerized and frustrated antiferromagnetic ladder system is studied by using a combination of numerical and analytical techniques. For the strongly frustrated regime, series expansions and bond operator techniques are employed to analyze zero magnetic field, whereas low-energy effective models are used to study the complete magnetization process. The interplay between frustration and dimerization gives rise to a rich plateau structure that is captured by effective models and corroborated by numerical density matrix renormalization group simulations, in particular, the emergence of intermediate plateaus at $M=\frac{1}{4}$ and $\frac{3}{4}$ of saturation in the magnetization curve is clarified.
\end{abstract}

DOI: 10.1103/PhysRevB.95.214426

\section{INTRODUCTION}

Antiferromagnetic spin ladders are paradigmatic examples of quantum magnetism in low dimensions [1-11]. These systems exhibit plenty of interesting properties, such as absence of long-range order, energy gaps, magnetic disordered phases, among others [12]. One particular issue of these systems that has captured both experimental and theoretical efforts is the existence of magnetization plateaus [13]. For a quantum spin- $S$ chain, the necessary condition for the occurrence of a magnetization plateau has been established by Oshikawa, Yamanaka, and Affleck (OYA) [14] as

$$
N(S-m) \in \mathbb{Z},
$$

where $N$ is the period of the spin state, $S$ the magnitude of spin, and $m$ the magnetization per site in units of $g \mu_{B}$. The OYA criterium has been successfully applied to different one-dimensional magnets $[9,15]$ and revisited by different techniques [16-20]. In this context, several quasione-dimensional compounds have been studied in the past years. As an example that motivates our work we mention the compound $\mathrm{Cu}_{2} \mathrm{Cl}_{4} \mathrm{D}_{8} \mathrm{C}_{4} \mathrm{SO}_{2}$, which presents a singlet ground state. In order to describe the properties of this material, different models have been proposed, ranging from tube-plaquette models [21,22], up to dimerized-zigzag ladder models [23]; the latter is similar to the model that we analyze in our work. Another system with a remarkable magnetic behavior is the $S=\frac{1}{2}$ zigzag ladder compound $\mathrm{NH}_{4} \mathrm{CuCl}_{3}$. In this material the magnetization curve presents two plateaus at $M=\frac{1}{4}$ and $\frac{3}{4}$ of the saturation magnetization, irrespective of the external field direction, but not at $M=0$ and $\frac{1}{2}$ [7].

In this paper, we study an antiferromagnet chain model in the zigzag geometry showing that the interplay between geometrical frustration and plaquette dimerization promotes the existence of plateaus at $M=\frac{1}{4}$ and $\frac{3}{4}$. Although we do not intend to provide a theoretical description of any particular material, our results may be relevant for the discussion of the emergence of rational magnetization plateaus and their magnon and spinon excitations in frustrated and dimerized zigzag ladders. The model analyzed in this work belongs to a family of frustrated antiferromagnetic systems with a fully dimerized exact ground state [24-26]. Recently, it was shown that by imposing certain exact condition to the couplings, the exact ground state of the system is robust against the inclusion of weak disorder [26]. This property provides to the model interesting magnetic properties when the unit cell is increased in order to consider plaquette dimerization. The exact condition can be imposed in one or both square plaquettes separately, giving a rich plateau structure, an aspect that will be exploited in this work.

The outline of the paper is as follows. In Sec. II we study the zero magnetic field phase diagram. Starting from the line where the ground state is exactly determined, we use a bond operator formalism to study the excitation gap. In Sec. III we analyze the effect of an external magnetic field on the model. We study the magnetization process by means of density matrix renormalization group (DMRG) calculations and exploring the parameters space we determine regions where different plateaus are present. In the highly dimerized plaquette regime, we derive low-energy effective models and studying the excitations we estimate the critical magnetic fields and the plateau widths. The interplay between dimerization and frustration results in a rich plateaus structure that is captured by the low-energy model and is consistent with the numerical results obtained through DMRG. Finally, in Sec. IV we present the conclusions, and some relevant formulas and expressions used throughout the work are displayed in the Appendix.

\section{ZERO MAGNETIC FIELD PHASE DIAGRAM}

\section{A. Exact ground state in the absence of magnetic field}

We consider the following Heisenberg model on a two-leg spin- $S$ ladder:

$$
\begin{aligned}
\mathbf{H}= & \sum_{n} J_{0}\left(\overrightarrow{\mathbf{S}}_{1, n} \cdot \overrightarrow{\mathbf{S}}_{2, n}+\overrightarrow{\mathbf{S}}_{3, n} \cdot \overrightarrow{\mathbf{S}}_{4, n}\right) \\
& +J\left(\overrightarrow{\mathbf{S}}_{1, n} \cdot \overrightarrow{\mathbf{S}}_{3, n}+\overrightarrow{\mathbf{S}}_{2, n} \cdot \overrightarrow{\mathbf{S}}_{4, n}\right)+J_{d} \overrightarrow{\mathbf{S}}_{2, n} \cdot \overrightarrow{\mathbf{S}}_{3, n} \\
& +K\left(\overrightarrow{\mathbf{S}}_{3, n} \cdot \overrightarrow{\mathbf{S}}_{1, n+1}+\overrightarrow{\mathbf{S}}_{4, n} \cdot \overrightarrow{\mathbf{S}}_{2, n+1}\right)+K_{d} \overrightarrow{\mathbf{S}}_{4, n} \cdot \overrightarrow{\mathbf{S}}_{1, n+1},
\end{aligned}
$$

where $\overrightarrow{\mathbf{S}}_{j, n}$ represents the $j$ th spin on the unit cell $n$. This ladder is schematized in Fig. 1. Following the lines sketched in Ref. [25], we can show that, provided the conditions $J_{d}=2 \mathrm{~J}$ and $K_{d}=2 K$, the ground state of the system corresponds to 


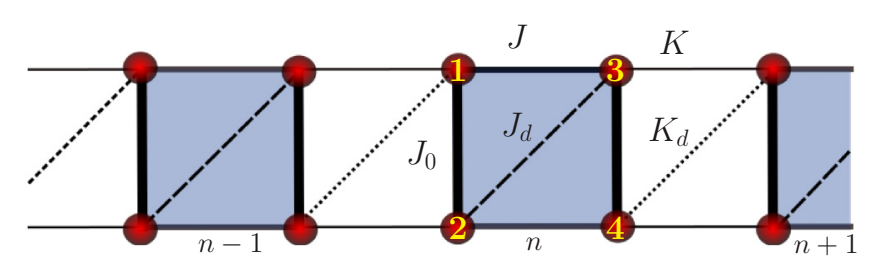

FIG. 1. Schematic representation of the plaquette dimerized zigzag ladder considered in this work. The red circles indicate spin- $S$ (particularized to $S=\frac{1}{2}$ for the rest of the work) and all couplings are antiferromagnetic.

the fully dimerized state $|\psi\rangle=\bigotimes_{i=1}^{N_{r}}|s\rangle_{i}$, with

$$
|s\rangle_{i}=\frac{1}{\sqrt{2 S+1}} \sum_{m=-S}^{S}(-1)^{m+S}|m,-m\rangle_{i},
$$

where the index $i$ labels the rungs in the ladder and $N_{r}$ is the number of rungs. The state $|m,-m\rangle_{i}$ is a product state such that $\mathbf{S}_{2 i-1, n}^{z}|m,-m\rangle_{i}=-\mathbf{S}_{2 i, n}^{z}|m,-m\rangle_{i}=$ $m|m,-m\rangle_{i}$ where the index $i=1,2$ corresponds to the two rungs in the unit cell $n$. This state remains the ground state of the system in the range of couplings $J<J_{0} / 2$ and $K<J_{0} / 2$. For this purpose, it is convenient to rewrite the Hamiltonian in the case $J_{d}=2 J, K_{d}=2 K$ as follows:

$$
\mathbf{H}=\sum_{n}\left[\mathbf{h}_{J}(n)+\mathbf{h}_{K}(n)\right],
$$

where $\mathbf{h}_{J}(n)$ and $\mathbf{h}_{K}(n)$ are Hamiltonians of the square plaquettes on unit cell $n$, given by

$$
\begin{aligned}
\mathbf{h}_{J}(n)= & \frac{J}{2}\left[\left(\overrightarrow{\mathbf{S}}_{1, n}+\overrightarrow{\mathbf{S}}_{2, n}+\overrightarrow{\mathbf{S}}_{3, n}\right)^{2}+\left(\overrightarrow{\mathbf{S}}_{2, n}+\overrightarrow{\mathbf{S}}_{3, n}+\overrightarrow{\mathbf{S}}_{4, n}\right)^{2}\right] \\
& +\frac{\left(J_{0}-2 J\right)}{4}\left[\left(\overrightarrow{\mathbf{S}}_{3, n}+\overrightarrow{\mathbf{S}}_{4, n}\right)^{2}+\left(\overrightarrow{\mathbf{S}}_{1, n}+\overrightarrow{\mathbf{S}}_{2, n}\right)^{2}\right] \\
& -\frac{3}{4}\left(J_{0}+J\right), \\
\mathbf{h}_{K}(n)= & \frac{K}{2}\left[\left(\overrightarrow{\mathbf{S}}_{3, n}+\overrightarrow{\mathbf{S}}_{4, n}+\overrightarrow{\mathbf{S}}_{1, n+1}\right)^{2}\right. \\
& \left.+\left(\overrightarrow{\mathbf{S}}_{4, n}+\overrightarrow{\mathbf{S}}_{1, n+1}+\overrightarrow{\mathbf{S}}_{2, n+1}\right)^{2}\right] \\
& +\frac{\left(J_{0}-2 K\right)}{4}\left[\left(\overrightarrow{\mathbf{S}}_{3, n}+\overrightarrow{\mathbf{S}}_{4, n}\right)^{2}+\left(\overrightarrow{\mathbf{S}}_{1, n}+\overrightarrow{\mathbf{S}}_{2, n}\right)^{2}\right] \\
& -\frac{3}{4}\left(J_{0}+K\right) .
\end{aligned}
$$

In this way, is easy to see that $\mathbf{H}$ is a semidefinite positive operator if $J<J_{0} / 2$ and $K<J_{0} / 2$. In the case $S=\frac{1}{2}$, that we will consider in this work, the energy per square plaquette is bounded from below by

$$
\begin{aligned}
\frac{E_{g s}}{N_{s}} \geqslant & \min _{l_{1,2}, l_{3,4}=0,1}\left\{-\frac{3}{4}\left(J_{0}+J\right)-\frac{3}{4}\left(J_{0}+K\right)\right. \\
& +\frac{J}{2} \sum_{n=1}^{2}\left[\left|\frac{1}{2}-l_{2 n-1,2 n}\right|\left(\left|\frac{1}{2}-l_{2 n-1,2 n}\right|+1\right)\right] \\
& +\frac{\left(J_{0}-2 J\right)}{4} \sum_{n=1}^{2}\left[l_{2 n-1,2 n}\left(l_{2 n-1,2 n}+1\right)\right]
\end{aligned}
$$

$$
\begin{aligned}
& +\frac{K}{2} \sum_{n=1}^{2}\left[\left|\frac{1}{2}-l_{2 n-1,2 n}\right|\left(\left|\frac{1}{2}-l_{2 n-1,2 n}\right|+1\right)\right] \\
& \left.+\frac{\left(J_{0}-2 K\right)}{4} \sum_{n=1}^{2}\left[l_{2 n-1,2 n}\left(l_{2 n-1,2 n}+1\right)\right]\right\},
\end{aligned}
$$

where $l_{1,2}\left(l_{3,4}\right)$ is the total spin in the rung between sites 1 and 2 (3 and 4 ). It is straightforward to see that the minimum value corresponds to $l_{1,2}=l_{3,4}=0$, given the bounding condition

$$
\frac{E_{g s}}{N_{s}} \geqslant-\frac{3}{2} J_{0} .
$$

To conclude, writing the Hamiltonian in terms of local operators on each rung

$$
\begin{array}{ll}
\overrightarrow{\mathbf{L}}_{1,2}(n)=\overrightarrow{\mathbf{S}}_{1, n}+\overrightarrow{\mathbf{S}}_{2, n}, & \overrightarrow{\mathbf{L}}_{3,4}(n)=\overrightarrow{\mathbf{S}}_{3, n}+\overrightarrow{\mathbf{S}}_{4, n}, \\
\overrightarrow{\mathbf{K}}_{1,2}(n)=\overrightarrow{\mathbf{S}}_{1, n}-\overrightarrow{\mathbf{S}}_{2, n}, & \overrightarrow{\mathbf{K}}_{3,4}(n)=\overrightarrow{\mathbf{S}}_{3, n}-\overrightarrow{\mathbf{S}}_{4, n}
\end{array}
$$

is easy to see that if we restrict the couplings to satisfy the condition $J_{d}=2 J$ and $K_{d}=2 K$, the state $|\psi\rangle$ is an eigenstate with eigenvalue $E=-\frac{3}{2} J_{0} N_{s}$. Therefore, we have proved that the state $|\psi\rangle$ is the ground state of the system along the lines $J_{d}=2 J$ and $K_{d}=2 K$ as long as the couplings satisfy $J<$ $J_{0} / 2$ and $K<J_{0} / 2$. Notice that these two last constraints represent a sufficient condition for the existence of this singlet ground state, but the actual range of couplings can be larger.

This exact result is a very convenient starting point to perform dimer expansions. Notice that the existence of a line where the singlet product state is the true ground state of the system allows us to use dimer expansions around a point in the parameter space where the Hamiltonian is maximally frustrated. In the following section we present some results obtained by different techniques based on dimer expansions.

\section{B. Bond operator approach}

Close to the line in the parameter space where the ground state corresponding to Hamiltonian (2) is exactly known, it is convenient to exploit the description in terms of bosonic operators, the so-called bond operators (BO) [27] which label the dimer's singlet-triplet spectrum. On the exact line the ground state is a direct product of states on each square plaquette at site $n$, consisting of a product of a singlet between spins located at sites $1, n$ and 2,n and another singlet between spins at $3, n$ and $4, n$. Within BO theory, the four spins $\vec{S}_{i}$ on each square plaquette are expressed as

$$
\begin{aligned}
& S_{\substack{1, n \\
2, n}}^{\alpha}=\frac{1}{2}\left( \pm s_{A, n}^{\dagger} a_{\alpha, n} \pm a_{\alpha, n}^{\dagger} s_{A, n}-\sum_{\beta, \gamma} i \varepsilon_{\alpha \beta \gamma} a_{\beta, n}^{\dagger} a_{\gamma, n}\right), \\
& S_{\substack{3, n \\
4, n}}^{\alpha}=\frac{1}{2}\left( \pm s_{B, n}^{\dagger} b_{\alpha, n} \pm b_{\alpha, n}^{\dagger} s_{B, n}-\sum_{\beta, \gamma} i \varepsilon_{\alpha \beta \gamma} b_{\beta, n}^{\dagger} b_{\gamma, n}\right),
\end{aligned}
$$


where $s_{A, n}^{(\dagger)}$ and $a_{\alpha, n}^{(\dagger)}$ destroy (create) the singlet and triplet states of the dimer between sites $1, n$ and $2, n$ and Greek labels, $\alpha=1,2,3$, refer to the threefold triplet multiplet. Equivalently, operators $s_{B, n}^{(\dagger)}$ and $b_{\alpha, n}^{(\dagger)}$ act on the dimer between sites $3, n$ and $4, n$. A hard-core constraint

$$
\begin{aligned}
& s_{A, n}^{\dagger} s_{A, n}+\sum_{\alpha} a_{\alpha, n}^{\dagger} a_{\alpha, n}=1, \\
& s_{B, n}^{\dagger} s_{B, n}+\sum_{\alpha} b_{\alpha, n}^{\dagger} b_{\alpha, n}=1
\end{aligned}
$$

is implied on each sublattice, which renders the algebra of the right-hand sides of Eqs. (10) and (11) identical to that of spins. Inserting the $\mathrm{BO}$ representation into a spin model leads to an interacting Bose gas. In the BO-MFT, singlets are condensed by $s^{(\dagger)} \rightarrow s \in$ Re and the constraint in the number of bosons per site is satisfied on average with a global Lagrange multiplier $\lambda$. In this approach, terms only up to second order in the BOs are retained and the quadratic Hamiltonian can be diagonalized by standard Bogoliubov transformation, leading to an energy E per unit cell of

$$
E=-\frac{3}{4} J_{0}-\frac{3}{2} s^{2} J_{0}+\left(5-2 s^{2}\right) \lambda+\frac{3}{N} \sum_{k}\left(\omega_{k}^{+}+\omega_{k}^{-}\right),
$$

with the triplon dispersion

$$
\omega_{k}^{ \pm}=|a| \sqrt{1 \pm|g| \frac{s^{2}}{a} \beta_{\delta}(k)},
$$

where

$$
\beta_{\delta}(k)=\frac{1}{2} \sqrt{1+\delta^{2}+2 \delta \cos (k)},
$$

$a=\frac{J_{0}}{4}-\lambda, g=J_{d}-2 J, \quad$ and $\quad s=\left\langle s_{A, n}\right\rangle=\left\langle s_{A, n}^{\dagger}\right\rangle=$ $\left\langle s_{B, n}\right\rangle=\left\langle s_{B, n}^{\dagger}\right\rangle$ is a real parameter and we have restricted the study to the case $K=\delta J$ and $K_{d}=\delta_{d} J_{d}$ with $\delta=\delta_{d}$, i.e., homogeneous dimerization. The general case will be analyzed with other techniques in the following sections. In order to obtain the mean field parameters $s$ and $a$ the energy $E$ is extremized, obtaining two self-consistency equations $\partial E / \partial a=0$ and $\partial E / \partial s=0$. These two equations can be combined, obtaining

$$
\begin{aligned}
& d=\frac{5}{2 J_{0}}-\frac{3}{8 \pi J_{0}} \operatorname{sign}(a) \int d k\left(\frac{1}{\gamma_{-}(k)}+\frac{1}{\gamma_{+}(k)}\right), \\
& a=J_{0}-\frac{3}{16 \pi}|g| \operatorname{sign}(a) \int d k\left(\frac{\beta_{\delta}(k)}{\gamma_{+}(k)}-\frac{\beta_{\delta}(k)}{\gamma_{-}(k)}\right),
\end{aligned}
$$

where $\gamma_{ \pm}(k)=\sqrt{1 \pm d|g| \beta_{\delta}(k)}$ and $d=s^{2} / a$. These equations can be solved self-consistently.

We mention, in passing, that the trivial limit, i.e., $g=0$, leads to $d=1 / J_{0}, s=1$, and $\lambda=-\frac{3}{4} J_{0}$, and therefore to a singlet-triplet gap of $\Delta=J_{0}$ and a ground-state energy of $E / N=-\frac{3}{2} J_{0}$, which is consistent with two saturated singlets per unit cell.

\section{Triplet dispersion and energy gap}

In this section we will analyze the triplet dispersion in absence of an external magnetic field in the model given by

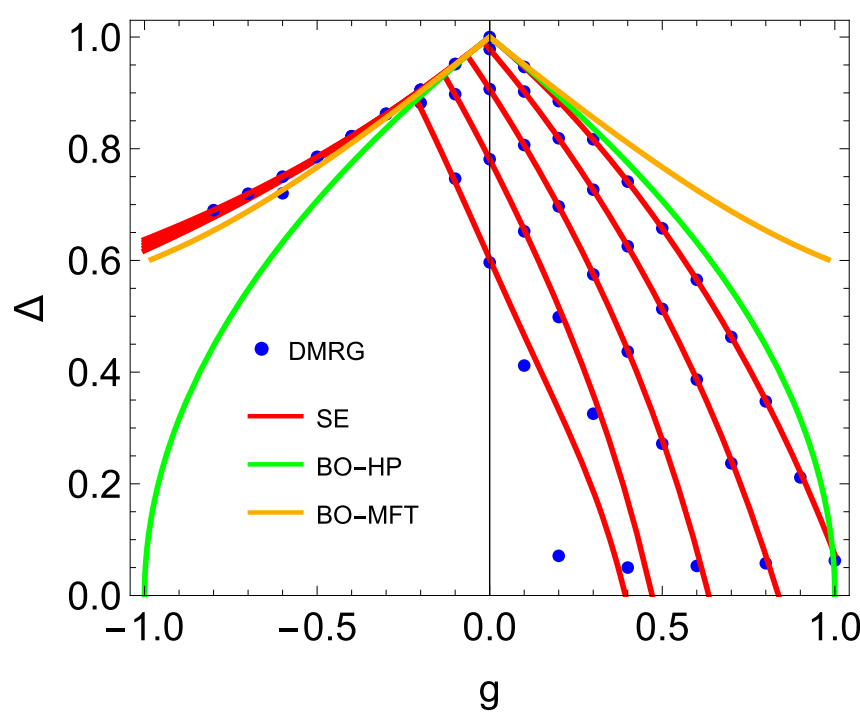

FIG. 2. Spin gap $\Delta$ versus $g=J_{d}-2 J$ obtained by means of different techniques employed in the work. The SE (red lines) and DMRG (blue dots) results are parametrized for $J=0,0.1,0.2,0.3$, and 0.4 from right to left. Notice that for bond operator approximations, Holstein-Primakoff (BO-HP, green line) and self-consistent (BO-MFT, orange line), the spin gap (and the dispersion) depend only on $g$, which is an artifact of the approximation. The peak shape of the gap maxima indicates a crossing of levels between $k=0$ (right) and $k=\pi$ (left) dispersion branches, detected by SE. Gap right branches together with the peaks shift to the left by increasing $J$ values and tend to collapse into a single $g$-dependent line for $g<0$, where the methods (except BO-HP) exhibit a very good agreement.

Eq. (2). For this, we will compare the results obtained by applying techniques of different nature, which complement each other and bring a perspective that none of the techniques can provide separately. The methods used here are on the one hand the numerical technique DMRG, and on the other hand strong coupling dimer and plaquette expansions, as the BO method from the previous section, and the series expansion technique (SE) considered in the Appendix.

Figure 2 summarizes the main result of this section, obtained by the different techniques mentioned previously, for the triplet gap, i.e., the minimum of the dispersion, as a function of the parameter $g=J_{d}-2 J$. Hereafter, all couplings are expressed in units of $J_{0}$ (which is set to unity). The case considered in this figure is that of a homogeneous structure without dimerization, i.e., $\delta=\delta_{d}=1$. The DMRG results, shown with blue dots in Fig. 2, for $J=0,0.1,0.2,0.3$, and 0.4 from right to left, and were calculated on finite structures of $L=40$ plaquettes (number of sites $N_{s}=160$ ) with periodic boundary conditions (PBC), maintaining up to $M=520$ states in the computation, which has shown to be enough to achieve the required precision. Unless specified otherwise, in the remainder of the work these will be the DMRG parameters used. All the DMRG computations presented in this work were performed with the open-source code ALPS [28].

On the other hand, the SE results are indicated by red line in this figure with the same $J$ parametrization as DMRG, 
and were obtained from an expansion in dimers to $O(10)$ by using the method of continuous unitary transformations (CUT) [29]. Finally, the BO results are of two types. The first one is the Holstein-Primakoff approximation (BO-HP), which is indicated by the green line in the Fig. 2. In the BO-HP, the self-consistent parameters in Eqs. (17) and (18) are solved for $g=0$ (obtaining $s=a=d=1$ ) and are replaced in the dispersion (15) which, after the rescaling $k \rightarrow 2 k$, imposed by halving the unit cell (dimer basis), takes the simple form $\omega_{\mathrm{HP}}=\sqrt{1-|g| \cos k}$. Note that this expression coincides with the treatment of the model under the random phase approximation (RPA) method [30]. The second type of $\mathrm{BO}$ result is the self-consistent solution of mean-field $\mathrm{BO}$ [Eqs. (17) and (18)], and is indicated by the orange line in Fig. 2.

Several comments are in order. First of all note that, apart from the $g$ dependence exists a residual interaction, represented here through the $J$ parametrization, that is captured by SE and DMRG, but not by BO. That is, BO encapsulates the entire dependence on $J$ and $J_{d}$ in $g$, which is an artifact of the mean field approximation. In addition, as can be observed, there is a very good quantitative agreement between DMRG and SE results, which is maintained until intermediate values of $J$, according to the perturbative character of the SE approximation.

On the other hand, note that the absolute maximum of the gap $(=1)$ at $g=0$, as well as the other maxima in Fig. 2, are nonderivable (peaks), representing a crossing of the dispersion branches at $k=0$ (right) and $k=\pi$ (left) of the peak, which are detected by the SE. This in turn reflects a change from a dimerized chain type to ladder type of spectrum, respectively.

Regarding the role of the residual interaction, it shifts the gap peak to the left $(g<0)$. However, its effect on the two dispersion branches is very different. While the branch $k=0$ (to the right of the peak) moves along with the peak to the left by increasing $J$, the branch $k=\pi$ on the left tends to collapse on a single curve, i.e., its dependence on $J$ and $J_{d}$ is captured mainly by $g$. These features are detected by means of SE and numerically corroborated by DMRG as can be seen in Fig. 2.

Finally, with respect to $\mathrm{BO}$, it is worth noting that although the mean field level does not take into account the residual interaction, but only $g$, it is able to capture some essential aspects of the gap behavior, not only qualitatively, but also up to a certain quantitative level. Here, the two approaches BO describe the gap with different precision on both sides of the gap peak. As can be seen in Fig. 2, the BO-HP (green line) adequately describes the right branch of the gap (in particular the $J=0$ line), while the BO-MFT (orange line) agreement for the left branch is very good, as compared with SE and DMRG results. This tendency of the dispersions to collapse with a single $g$ dependence for $g<0$ could be related to the fact that in that region the gap remains large, i.e., more adiabatically connected to the exact line $g=0$, as shown in the next section through a sweep in the $J-J_{d}$ plane of the gap. Finally, let us mention that in quantitative terms, the role of the residual interaction and the dependence on $g$ is clarified in the Appendix, where it is explicitly shown that at leading order the dispersions obtained by SE and BO-HP coincide and depend only on $g$.

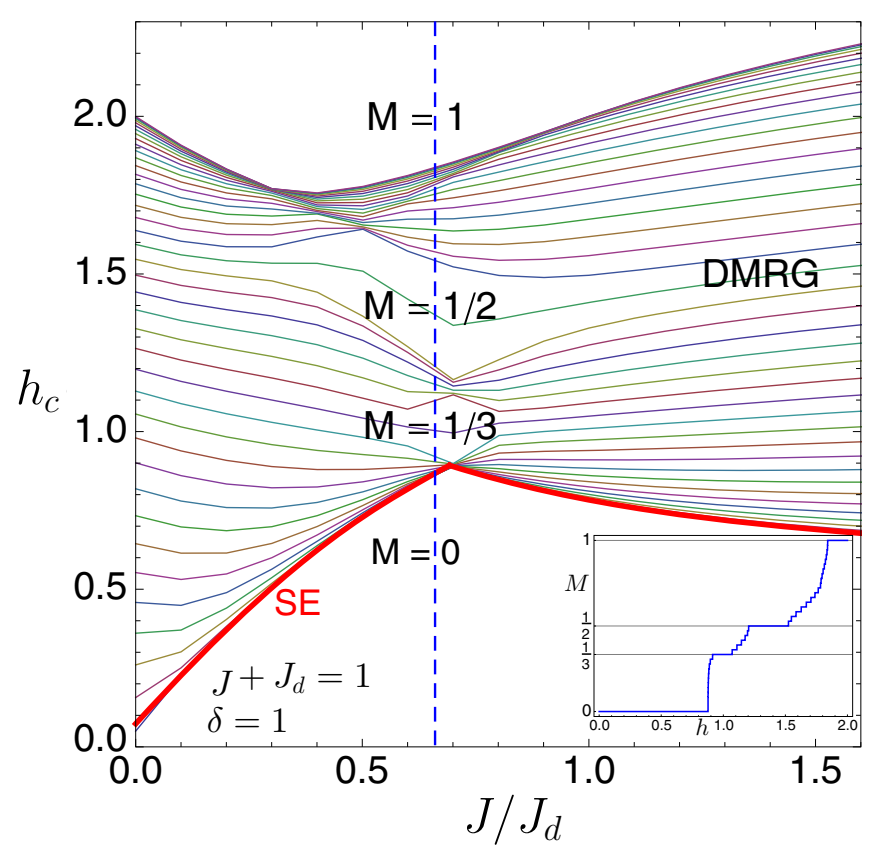

FIG. 3. Critical fields (thin lines) delimiting the different magnetization sectors on a finite system, determined by DMRG, as a function of $J / J_{d}$, along the line $J+J_{d}=1$. Note the presence of two intermediate plateaus at $M=\frac{1}{3}$ and $\frac{1}{2}$. The bold red line indicates $M=0$ critical line (in thermodynamic limit), where SE triplet gap vanishes, showing an excellent agreement with the corresponding DMRG determination. Inset: magnetization curve for $J=0.4$ and $J_{d}=0.6$ (i.e., $J / J_{d}=\frac{2}{3}$ ). This corresponds to the dashed blue line in the main figure.

\section{MAGNETIC FIELD PHASES}

\section{A. Plateau phases without plaquette dimerization}

In this section we will analyze the effect of an external magnetic field on the model given by Eq. (2). To simplify the analysis, we will start by studying the case of homogeneous frustrated plaquettes, i.e., $\delta=\delta_{d}=1$. Although this case has been previously studied in different regions of the space of parameters and employing different techniques [31-36], our analysis presents it in a unified way and contextualizes our subsequent study of the general case with frustration and dimerization between plaquettes of the following sections.

As it is known the presence of a magnetic field opens the possibility of the emergence of plateau phases in the magnetization curves, where the magnetization is fixed at a certain value, for a finite interval of the magnetic field.

In Fig. 3, we show the critical fields $h_{c}$ delimiting the different possible values of magnetization, determined by DMRG, in function of $J / J_{d}$, along the line $J+J_{d}=1$. The choice of the parameters in this figure is in order to show in a single representation the emergence of intermediate plateaus. As can be observed, apart from the zero field $(M=0)$ and saturation $(M=1)$ plateaus, two additional plateaus at $M=\frac{1}{3}$ and $\frac{1}{2}$ are present. Note that the critical line crossing each of these two plateaus represents a small jump that is an artifact due to the open boundary conditions used for the computation.

Additionally, in Fig. 3, the critical line delineating the plateau at $M=0$, obtained by the SE triplet gap closure 
condition, is presented with red line. The peak of this curve represents the crossing of the gap closing branches at $k=0$ and $\pi$. As can be observed, the quantitative agreement between DMRG and SE for the line $M=0$ is excellent throughout the range of parameters shown in the figure.

On the other hand, Fig 4 shows the extension of the plateaus at $M=0(\mathrm{a}), \frac{1}{3}$ (b), and $\frac{1}{2}$ (c) in the plane $J-J_{d}$ calculated on finite systems with the DMRG technique. The determination is not intended to be quantitatively accurate, for which an analysis of a finite-size scaling that goes beyond the objectives of the work should be carried out, but rather to determine the regions in the parameter space where the plateaus are most prominent.

Let us first consider Fig. 4(a) where the width of $M=0$ plateau is considered. The largest plateau width $(\Delta=1)$ is at the origin, corresponding to the limiting case of isolated dimers (see Fig. 1). The maximum width of the plateau follows the line of maximum frustration, $g=0$, indicated in this figure with a dotted blue line, up to $J \simeq 0.2$, beyond which deviations towards $g<0$ become increasingly marked. In the lower right inset of Fig. 4(a) the SE triplet gap is depicted showing the same trend as the DMRG determination. This deviation is consistent with the observed shift of the gap peak in Fig. 2, predicted by SE and DMRG, as well as the tendency to collapse into a single $g$-dependent triplet gap curve for $g<0$. Another particular case of our model is represented by the point ( $\left.J=0, J_{d}=1\right)$, corresponding to the homogeneous Heisenberg chain, which is gapless. Note that both DMRG and SE predict a tendency toward the gap closure (dark zone) approaching that point. The model remains gapless along the line $J_{d}=1$ up to the point ( $J \simeq 0.241, J_{d}=1$ ) [37], indicated by a green circle in Fig. 4(a). From this point the system is gapped and for $\left(J=0.5, J_{d}=1\right.$ ) (Majumdar-Gosh point) the model displays the exact dimer-product zero field ground state, which extends all over the line $g=0$ analyzed in Sec. II A.

Regarding the $M=\frac{1}{3}$ plateau, Fig. 4(b) shows that this plateau emerges in a certain reduced and highly frustrated chain limit of the model, where $J, J_{d}$, are of the order of the unity, reaching values of plateau width of $\approx 0.6$. The nature of this plateau is completely different to the others analyzed in this work, having a classical origin [38], characterized by an "up-up-down" ordering which stabilizes this magnetization value. This state adiabatically evolves from the Ising chain with first- and second-neighbor interactions, and survives up to the Heisenberg isotropic limit. This plateau has been extensively analyzed in the works of Refs. [39-42]. In addition, the techniques of strong plaquette expansions that we will use (see next section) are not especially suitable to treat this plateau since it emerges in a region where all the couplings are of the same order. For these reasons, we will not elaborate more on the same in this work.

Finally, Fig. 4(c) depicts the extension of the $M=\frac{1}{2}$ plateau determined by DMRG. The emergence of this plateau has been analyzed in terms of a dimerized and frustrated ladder from a numerical point of view $[42,43]$. The dashed yellow lines indicate estimations of high field $(h>1)$ limit of $M=\frac{1}{2}$ phase plateau, from an effective low-energy dimer model description of the system [44,45], indicating a similar trend to our findings.

In the following sections, we will develop a low-energy plaquette effective model, which will account for present plateaus
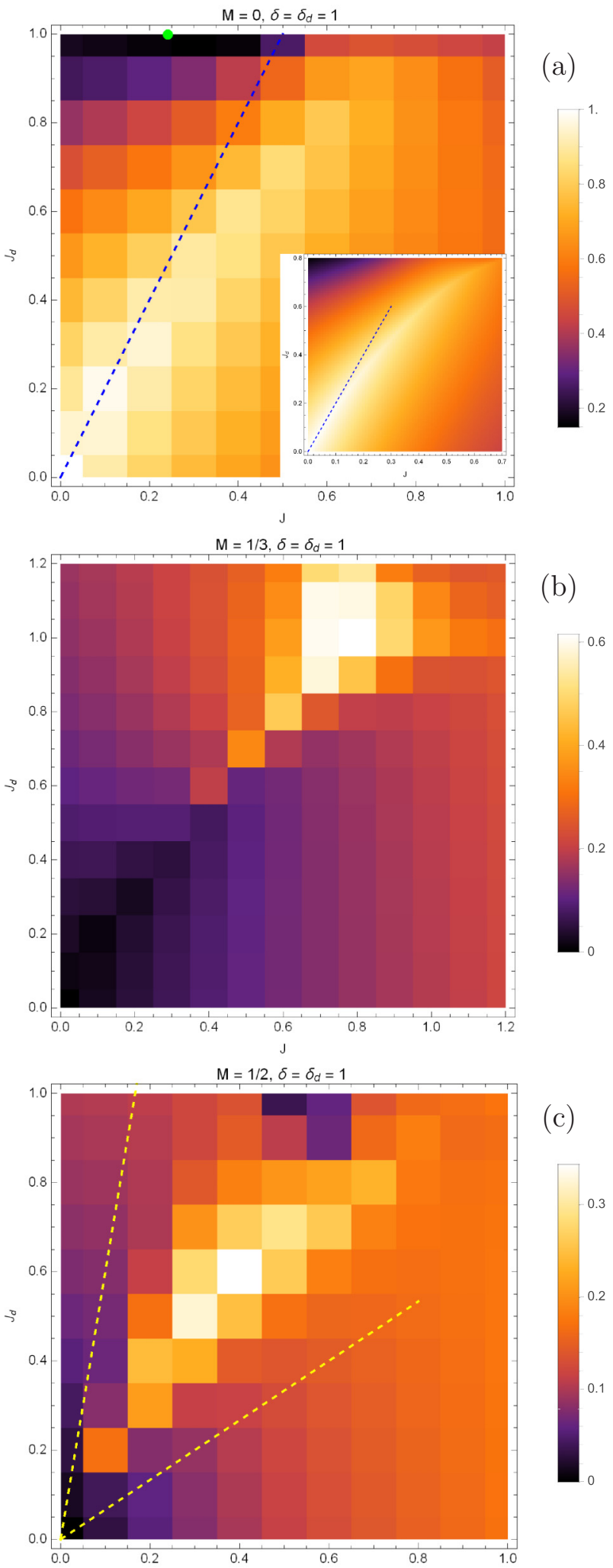

FIG. 4. Phase diagrams of the plateaus at $M=0, \frac{1}{3}$, and $\frac{1}{2}$ (top to bottom) for $\delta=\delta_{d}=1$ in the plane $J-J_{d}$, up values of the order of one, analyzed by means of DMRG. The color indicates a decreasing plateau width (from white to black). The blue dotted line in the top panel indicates the line of maximum frustration $g=0$. In the inset of (a) the SE triplet gap is depicted, showing good agreement with the DMRG determination. 
(except $M=\frac{1}{3}$, which would require higher-order terms) and predict the emergence of others at $M=\frac{1}{4}$ and $\frac{3}{4}$ due to the interplay between frustration and interplaquette dimerization.

\section{B. Effective model description of dimerized plaquette Hamiltonian}

In the previous sections we have considered the frustrated homogeneous plaquette case, where the frustration degree is controlled by $g=J_{d}-2 J$, being maximal along the line $g=$ 0 , where the model exhibits an exact rung-dimer product state. In this section, we will explore the effect of plaquette dimerization via the parameters $\delta=K / J$ and $\delta_{d}=K_{d} / K$. The interplay between on-plaquette frustration and interplaquette dimerization gives rise to a richer plateau structure that we will analyze in a weak interacting plaquette regime, starting from the limit of decoupled plaquettes. It is worth mentioning that an equivalent model, although with a different parametrization, has been studied analytically using a field theory bosonization approach [46]. This study, however, is complementary to ours, since it starts from the strong chain limit.

We start by analyzing the Hilbert space corresponding to isolated plaquettes, which is spanned by a basis of 16 states containing 2 singlets, 9 triplets, and 5 quintuplets, which we identify generically by $\left|s_{i}\right\rangle,\left|t_{j}\right\rangle$, and $\left|q_{k}\right\rangle$, respectively. These states are listed in the Appendix A (Table I). On each plaquette in the absence of magnetic field $h$ the ground state corresponds to the singlet $\left|s_{0}\right\rangle$. When the magnetic field is turned on, the energy corresponding to the singlet and a triplet becomes closer until a critical value where a level crossing occurs and a triplet becomes the ground state. By further increasing the magnetic field we obtain a second level crossing between triplet and the quintet state $\left|q_{0}\right\rangle$. Here, the local structure of the plaquette couplings becomes important. According to the values of the internal couplings, two different types of triplets are involved in the crossings, depending on whether $J_{d}<\frac{2 J}{1+J}$ or $J_{d}>\frac{2 J}{1+J}$. In the following we refer these two different scenarios as cases $A$ and $B$, respectively. In the case $A(B)$ the singlet state $\left|s_{0}\right\rangle$ is degenerate with a triplet state $\left|t_{A(B)}\right\rangle$ in the first transition, at the critical field $h c_{1 A(B)}$, whereas $\left|t_{A(B)}\right\rangle$ is degenerate with the quintet state $\left|q_{0}\right\rangle$ in the second transition at the critical field $h c_{2 A(B)}$. These possibilities are represented schematically in Eq. (19):

$$
\begin{aligned}
& \left|s_{0}\right\rangle \stackrel{h c_{1 A}}{\longrightarrow}\left|t_{A}\right\rangle \stackrel{h c_{2 A}}{\longrightarrow}\left|q_{0}\right\rangle, \quad J_{d}<\frac{2 J}{1+J}, \\
& \left|s_{0}\right\rangle \stackrel{h c_{1 B}}{\longrightarrow}\left|t_{B}\right\rangle \stackrel{h c_{2 B}}{\longrightarrow}\left|q_{0}\right\rangle, \quad J_{d}>\frac{2 J}{1+J} .
\end{aligned}
$$

Around these level crossing points we can derive an effective model. Let us write the Hamiltonian as follows:

$$
\mathbf{H}_{\mu}=\mathbf{H}_{0, \mu}+\mathbf{H}_{\text {int }, \mu},
$$

where the extra index $\mu$ indicates the possible cases mentioned, i.e., $\mu=\{1 A, 2 A, 1 B, 2 B\}$, being

$$
\begin{aligned}
\mathbf{H}_{0, \mu}= & \sum_{n}\left[\left(\overrightarrow{\mathbf{S}}_{1, n} \cdot \overrightarrow{\mathbf{S}}_{2, n}+\overrightarrow{\mathbf{S}}_{3, n} \cdot \overrightarrow{\mathbf{S}}_{4, n}\right)\right. \\
& +J\left(\overrightarrow{\mathbf{S}}_{1, n} \cdot \overrightarrow{\mathbf{S}}_{3, n}+\overrightarrow{\mathbf{S}}_{2, n} \cdot \overrightarrow{\mathbf{S}}_{4, n}\right)+J_{d} \overrightarrow{\mathbf{S}}_{2, n} \cdot \overrightarrow{\mathbf{S}}_{3, n} \\
& \left.-h c_{\mu} \sum_{j=1}^{4} \overrightarrow{\mathbf{S}}_{j, n}\right] \\
\mathbf{H}_{i n t, \mu}= & \sum_{n}\left[K\left(\overrightarrow{\mathbf{S}}_{3, n} \cdot \overrightarrow{\mathbf{S}}_{1, n+1}+\overrightarrow{\mathbf{S}}_{4, n} \cdot \overrightarrow{\mathbf{S}}_{2, n+1}\right)\right. \\
& \left.+K_{d} \overrightarrow{\mathbf{S}}_{4, n} \cdot \overrightarrow{\mathbf{S}}_{1, n+1}-\left(h-h c_{\mu}\right) \sum_{j=1}^{4} \overrightarrow{\mathbf{S}}_{j, n}\right] .
\end{aligned}
$$

\begin{tabular}{|c|c|c|c|}
\hline$S_{T}$ & $S_{T}^{z}$ & & Energy \\
\hline \multirow[t]{6}{*}{0} & 0 & \multirow[t]{2}{*}{$\left|s_{0}\right\rangle$} & $-\frac{J}{2}-\frac{J_{d}}{4}-\frac{1}{2} \sqrt{4 J^{2}-2 J J_{d}-4 J+J_{d}^{2}-2 J_{d}+4}-\frac{1}{2}$ \\
\hline & 0 & & $-\frac{J}{2}-\frac{J_{d}}{4}+\frac{1}{2} \sqrt{4 J^{2}-2 J J_{d}-4 J+J_{d}^{2}-2 J_{d}+4}-\frac{1}{2}$ \\
\hline & -1 & $\left|t_{A}\right\rangle$ & $-h-\frac{J}{2}+\frac{J_{d}}{4}-\frac{1}{2}$ \\
\hline & -1 & $\left|t_{B}\right\rangle$ & $-h-\frac{J_{d}}{4}-\frac{1}{2} \sqrt{J^{2}-2 J+J_{d}^{2}+1}$ \\
\hline & -1 & & $-h-\frac{J_{d}}{4}+\frac{1}{2} \sqrt{J^{2}-2 J+J_{d}^{2}+1}$ \\
\hline & 0 & & $-\frac{J_{d}}{4}-\frac{1}{2} \sqrt{J^{2}-2 J+J_{d}^{2}+1}$ \\
\hline \multirow[t]{7}{*}{1} & 0 & & $-\frac{J_{d}}{4}+\frac{1}{2} \sqrt{J^{2}-2 J+J_{d}^{2}+1}$ \\
\hline & 0 & & $-\frac{J}{2}+\frac{J_{d}}{4}-\frac{1}{2}$ \\
\hline & 1 & & $h-\frac{J}{2}+\frac{J_{d}}{4}-\frac{1}{2}$ \\
\hline & 1 & & $h-\frac{J_{d}}{4}-\frac{1}{2} \sqrt{J^{2}-2 J+J_{d}^{2}+1}$ \\
\hline & 1 & & $h-\frac{J_{d}}{4}+\frac{1}{2} \sqrt{J^{2}-2 J+J_{d}^{2}+1}$ \\
\hline & -2 & \multirow[t]{5}{*}{$\left|q_{0}\right\rangle$} & $-2 h+\frac{J}{2}+\frac{J_{d}}{4}+\frac{1}{2}$ \\
\hline & -1 & & $-h+\frac{J}{2}+\frac{J_{d}}{4}+\frac{1}{2}$ \\
\hline \multirow[t]{3}{*}{2} & 0 & & $\frac{J}{2}+\frac{J_{d}}{4}+\frac{1}{2}$ \\
\hline & 1 & & $h+\frac{J}{2}+\frac{J_{d}}{4}+\frac{1}{2}$ \\
\hline & 2 & & $2 h+\frac{J}{2}+\frac{J_{d}}{4}+\frac{1}{2}$ \\
\hline
\end{tabular}

TABLE I. Eigenvalues corresponding to the 16 eigenstates of the plaquette. The four low-energy states involved in the perturbative calculation are labeled as $\left|s_{0}\right\rangle,\left|t_{A}\right\rangle,\left|t_{B}\right\rangle$, and $\left|q_{0}\right\rangle$. 
We construct the effective Hamiltonian via degenerate perturbation theory $[6,44,45,47,48]$

$$
\mathbf{H}_{\mathrm{eff}, \mu}=\mathbf{H}_{\mathrm{eff}, \mu}^{(1)}+\mathbf{H}_{\mathrm{eff}, \mu}^{(2)}+\cdots,
$$

where the superscript indicates the order of the perturbation, although for our purpose in this paper we will only consider the first order of the expansion.

Since at the crossing point the ground state of each plaquette is doubly degenerate (except for $J_{d}=\frac{2 J}{1+J}$ which is triply degenerate and will not be considered here), the original dimerized plaquette model with 16 states per site is reduced to an effective spin- $\frac{1}{2}$ chain model involving the two mentioned degenerate low-energy states at the crossover.

The first-order effective Hamiltonian around $h c_{\mu}$ is obtained by applying the standard degenerate perturbation theory

$$
\mathbf{H}_{\mathrm{eff}, \mu}^{(1)}=\sum_{i, j=1,2}\left|v_{i}\right\rangle\left\langle v_{i}\left|\mathbf{H}_{\mathrm{int}, \mu}\right| v_{j}\right\rangle\left\langle v_{j}\right|,
$$

where states $\left|v_{i}\right\rangle$ and $\left|v_{j}\right\rangle$ span the set $\left\{\left|s_{0}\right\rangle, \mid t_{A(B)\rangle}\right\}$ and $\left\{\left|q_{0}\right\rangle, \mid t_{A(B)\rangle}\right\}$ for $\mu=1 A(B)$ and $\mu=2 A(B)$, respectively. In this way is obtained, up to a constant term,

$$
\begin{aligned}
\mathbf{H}_{\mathrm{eff}, \mu}^{(1)}= & \sum_{n} J_{x y, \mu}\left(\mathbf{S}_{n}^{x} \cdot \mathbf{S}_{n+1}^{x}+\mathbf{S}_{n}^{y} \cdot \mathbf{S}_{n+1}^{y}\right) \\
& +J_{z z, \mu} \mathbf{S}_{n}^{z} \cdot \mathbf{S}_{n+1}^{z}-\tilde{h}_{\mu} \mathbf{S}_{n}^{z},
\end{aligned}
$$

where effective couplings $J_{x y, \mu}, J_{z z, \mu}$, and $\tilde{h}_{\mu}$ are functions of the original plaquette model couplings $J, J_{d}, K, K_{d}$, and $h$. The explicit expressions of these functions are available in Appendix A [Eqs. (A2)-(A15)]. In addition, the pseudo spin- $\frac{1}{2}$ operators in Eq. (24) are projectors of the degenerate plaquette basis

$$
\begin{aligned}
& \mathbf{S}_{n}^{z}=\frac{1}{2}\left(\left|t_{A(B)}\right\rangle\left\langle t_{A(B)}|-| s_{0}\right\rangle\left\langle s_{0}\right|\right)_{n}, \\
& \mathbf{S}_{n}^{\dagger}=\left(\left|s_{0}\right\rangle\left\langle t_{A(B)}\right|\right)_{n}, \quad \mu=1 A(B), \\
& \mathbf{S}_{n}^{z}=\frac{1}{2}\left(\left|q_{0}\right\rangle\left\langle q_{0}|-| t_{A(B)}\right\rangle\left\langle t_{A(B)}\right|\right)_{n}, \\
& \mathbf{S}_{n}^{\dagger}=\left(\left|t_{A(B)}\right\rangle\left\langle q_{0}\right|\right)_{n}, \quad \mu=2 A(B),
\end{aligned}
$$

where $\mathbf{S}_{n}^{\dagger}=\mathbf{S}_{n}^{x}+i \mathbf{S}_{n}^{y}$.

\section{Phase diagram of the spin- $\frac{1}{2}$ chain effective Hamiltonian}

The effective model given by Eq. (24) corresponds to an XXZ spin- $-\frac{1}{2}$ chain and can be solved exactly via the Bethe ansatz [49]. Here, we briefly review the main characteristics of the Bethe ansatz solution, considering the different phases present, and their implications in the magnetization process. For simplicity in this discussion we will simplify the notation of Eqs. (24) by removing supraindices and subindices and indicating the three principal parameters of the model as $J_{x y}, J_{z z}$, and the magnetic field $h$. The phase diagram in the plane $\left(\Delta=J_{z z} / J_{x y}, h / J_{x y}\right)$ is shown in Fig. 5(a). As can be observed, there are three phases: ferromagnetic ( $\mathrm{F}$, yellow), Néel (N, blue), and Luttinger liquid (LL, gray). The $\mathrm{F}$ and $\mathrm{N}$ phases are gapped, with spin-1 magnon and spin- $\frac{1}{2}$ spinon domain-wall type of excitations, respectively. On the other hand, the intermediate phase LL is gapless and exhibits (a)

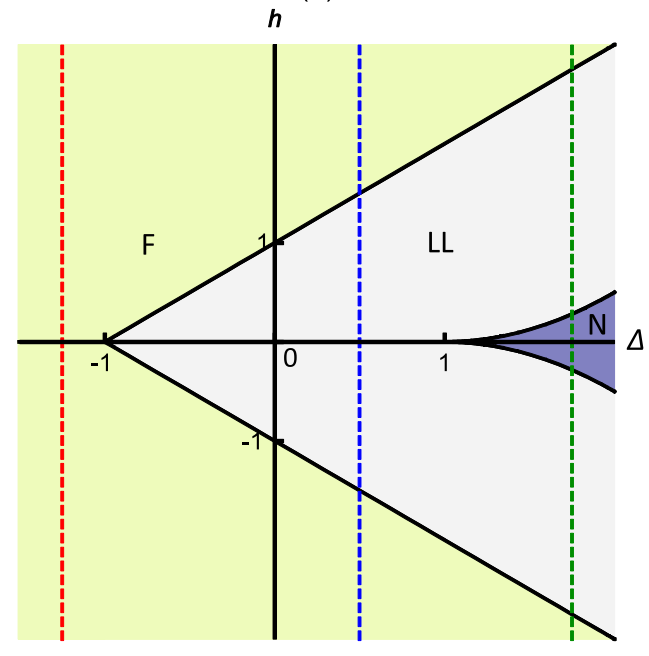

(b)

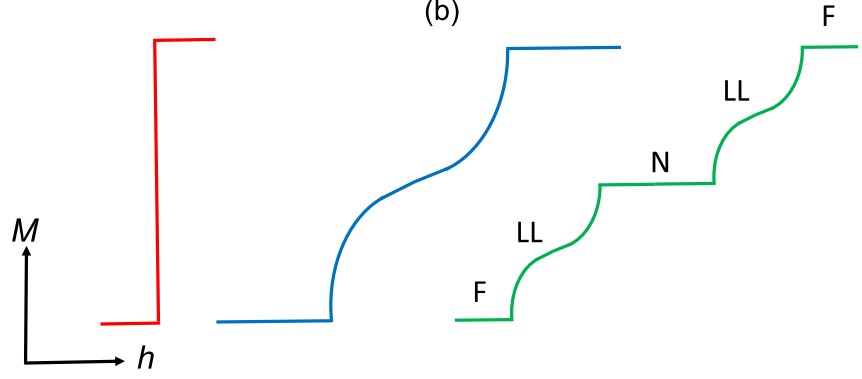

FIG. 5. (a) Phase diagram $h$ vs $\Delta$ (in units of $J_{x, y}$ ) of the XXZ spin- $\frac{1}{2}$ chain. The three phases present in the model are ferromagnetic ( $\mathrm{F}$, yellow), Néel ( $\mathrm{N}$, blue), and Luttinger liquid (LL, gray). The $\mathrm{F}$ and $\mathrm{N}$ phases are gapped, and exhibit spin-1 magnon and spin- $\frac{1}{2}$ spinon excitations, respectively, whereas LL phase is gapless and presents quasi-long-range order. (b) Schematic representation of the magnetization curves $M(h)$ corresponding to the three paths indicated with red, blue, and green dotted lines in (a). Note that each gapped phase translates into plateaus in the magnetization curves (see the text for details).

quasi-long-range order. Regarding critical lines in Fig. 5(a), the straight lines separating LL and $\mathrm{F}$ phases are explicitly given in terms of $\Delta$ by

$$
h_{\mathrm{L}-\mathrm{F}}= \pm(1+\Delta) \text {, }
$$

whereas the border between LL and $\mathrm{N}$ phase is obtained by solving [50]

$$
h_{\mathrm{L}-\mathrm{N}}= \pm \sinh (g) \sum_{n=-\infty}^{\infty} \frac{(-1)^{n}}{\cosh (n g)},
$$

where $g=\operatorname{arcosh} \Delta$. Note that both $h_{\mathrm{L}-\mathrm{F}}$ and $h_{\mathrm{L}-\mathrm{N}}$ are given in units of $J_{x y}$.

The different phases present in the model translate into distinctive characteristics in the magnetization curves. The gapped phases $\mathrm{F}$ and $\mathrm{N}$ exhibit a plateau in the magnetization curve, while in the LL phase the magnetization continuously increases with the magnetic field. This dependence is illustrated in Fig. 5. In Fig. 5(a), three paths with red, blue, 
and green dotted lines are indicated in the phase diagram, while in Fig. 5(b) the magnetization curves corresponding to these three paths are shown schematically. Note that the vertical axis in Fig. 5(a) is represented as horizontal axis in Fig. 5(b). The red curve represents the simplest case, where the system is always in the F phase and has a stepwise structure with two plateaus, jumping directly from one to another with the sign change of the magnetic field. The blue magnetization curve represents an intermediate case, in which the two plateaus corresponding to the $\mathrm{F}$ phase are connected through a region of continuous growth of the magnetization with the field, whose shape is characteristic of the LL phase. Finally, the green magnetization curve in Fig. 5(b) indicates the more general case, which comprises the three phases. In this case, in addition to the plateaus at the extremes due to the F phase, an additional intermediate plateau indicative of the $\mathrm{N}$ phase is present. These three plateaus are connected by LL phases, consistently with the path indicated in green in Fig. 5(a).

Before concluding with this section, we would like to comment on another case where the plateau structure has a simple stepped form. This case is the Ising limit of the model, in which $J_{x, y} \rightarrow 0(\Delta \rightarrow \infty)$, i.e., the right infinite end of Fig. 5(a). Here, the magnetization curve exhibits the three plateaus with direct jumps between them, without intermediate LL phase.

\section{Effective model description of magnetization process}

In this section we will analyze how the effective low-energy spin- $\frac{1}{2}$ chain model with anisotropy $\Delta$, obtained previously, captures the main aspects of the magnetization process of the original plaquette model in a certain, strong plaquette coupling, regime of the parameter space. The effective model is not only useful to account for the numerical results obtained through DMRG, but also brings a direct physical interpretation of the structure of plateaus that can emerge in the original model. In addition, it provides a tool that allows us to detect some characteristics, such as small intermediate plateaus, which would be difficult to detect by direct numerical scanning.

To apply the results of the effective model to the original plaquette model, note that according to Eq. (25), the effective pseudo-spin-operator eigenvalues $S_{z}=-\frac{1}{2}\left(\frac{1}{2}\right)$ correspond to plaquette $S_{z}=0(1)$, of the singlet $\left|s_{0}\right\rangle$ and triplet $\left|t_{A(B)}\right\rangle$ eigenvalues of the first level crossing $1 A(B)$. Therefore, the three possible magnetization plateaus in the effective chain model at $\{-1,0,1\}$ are mapped onto $\left\{0, \frac{1}{4}, \frac{1}{2}\right\}$ magnetization plateaus in the plaquette model, normalized to maximum spin per site and plaquette, respectively. Similarly for the second level crossing $2 A(B)$ between the triplet $\left|t_{A(B)}\right\rangle$ and quintet $\left|q_{0}\right\rangle$, Eq. (26) maps $\{-1,0,1\}$ onto $\left\{\frac{1}{2}, \frac{3}{4}, 1\right\}$, corresponding to effective chain and plaquette models, respectively.

Now, we will describe qualitatively the structure of the magnetization curves in terms of the effective models. To this end, Fig. 6 illustrates different representative plateau structures in the original model obtained by means of DMRG, shown with blue solid line. In particular, Fig. 6(a) shows magnetization curves with a single transition between type-F phases at zero magnetic field and saturation, intermediated by a LL phase. Note that the structure of small steps in the LL portion of the curve is due to the finite size of the numerical calculation. In
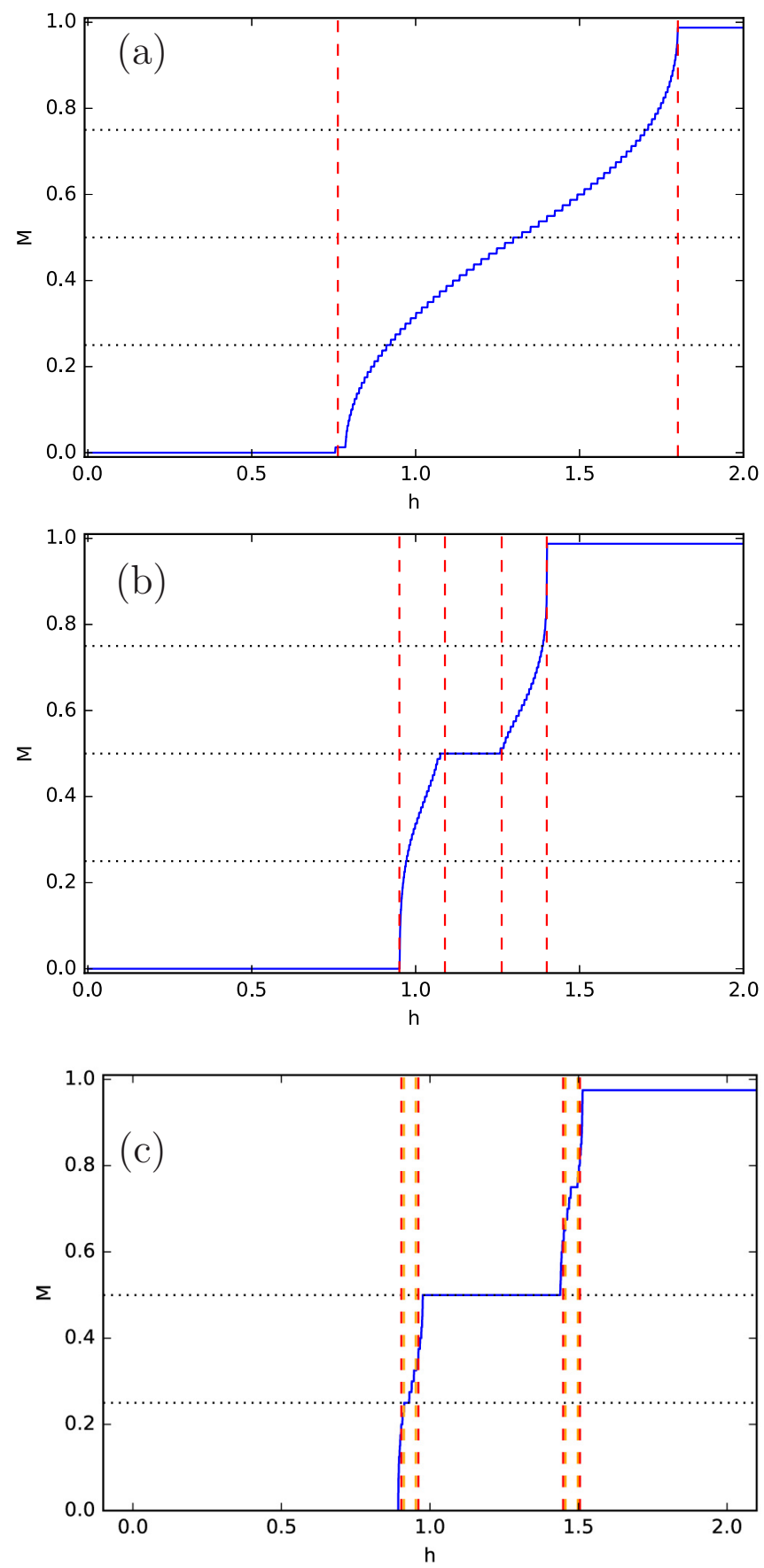

FIG. 6. Magnetization curves corresponding to $J=K=0.4$ and $J_{d}=K_{d}=0.3$ (a), $J=K=0.2$ and $J_{d}=K_{d}=0.3$ (b), $J=$ $0.8, J_{d}=0.6, K=0.2$, and $K_{d}=0.45$ (c). Red dashed lines and orange dashed lines correspond to critical fields obtained by means of magnon and spinon dispersions, respectively.

Fig. 6(b), an additional plateau emerges at midmagnetization. However, according to the previous analysis, this plateau is type $\mathrm{F}$, like the other two present here. The cases of Figs. 6(a) and 6(b) are not dimerized, i.e., $\delta=\delta_{d}=1$, and illustrate a general feature: in absence of dimerization between plaquettes, there can only be plateaus at $M=0, \frac{1}{2}$, and 1 in the magnetization curve, sharing spin-1 magnon excitations. The origin of these plateaus is intrinsic to each isolated plaquette, 
as a consequence of the two $[1 A(B), 2 A(B)]$ possible level crossings, which are only renormalized by the interaction between plaquettes.

On the other hand, Fig. 6(c) shows a plaquette-dimerized case, where additional plateaus at $M=\frac{1}{4}, \frac{3}{4}$ are present. These plateaus are indicative of the $\mathrm{N}$-type phase which, as we have mentioned, presents spinonlike excitations and is generated by the interaction between plaquettes. A necessary (but not sufficient) condition for their emergence is the presence of plaquette dimerization.

Regarding the size of type-N plateaus, we have observed that in general they are smaller than those of type F. For this reason, the effective model is very useful for their detection since numerically they could easily be overlooked, given the quite large number of parameters involved in the original model.

In addition, the effective model not only facilitates the detection of the intermediate N-type plateaus, but also highlights the role of frustration in the emergence of them. In fact, it is observed that apart from dimerization, type-N plateaus grow with frustration, being largest along the line of maximum frustration, i.e., $g=J_{d}-2 J=0$ and $g_{k}=K_{d}-2 K=0$, whenever this condition can be satisfied, which depends on the crossing levels ( $A$ or $B$ ) involved. It is interesting to note that in a certain range of validity frustration-induced type- $\mathrm{N}$ plateaus are maximal along the maximum frustration line, where in addition the original model [Eq. (1)] exhibits an exact dimer singlet-product zero field ground state. In some sense, it suggests that this condition is not only relevant at zero magnetic field, but also plays a role in the magnetization process.

Finally, regarding the dotted red and yellow vertical lines showed in the three panels of Fig. 6, they are quantitative estimations of critical fields delimiting the different plateaus based on the dispersion of magnon and spinon excitations of the effective model, and will be discussed in the next section.

\section{E. Magnetic excitations and critical fields}

Here, we will analyze the excitations on the plateau structures in the effective chain model, which in turn will allow us to obtain the critical fields, i.e., the borders of the plateaus and compare with the numerical DMRG results from the original model.

First, we consider the saturation plateaus at $-(+1)$ in the effective chain model. These correspond to ferromagnetic states, with all the effective spins on each site polarized along the negative and positive directions of the effective field, respectively. The elemental magnon excitation consists of a spin-wave composed by a linear combination of states with a local one-spin inversion, carrying $\Delta S_{z}=+(-1)$. The application of the effective Hamiltonian [Eq. (24)] on this state (subtracting the ground state) gives rise, by a standard calculation, to the magnon $(M)$ dispersion

$$
\omega_{M, \mu}^{ \pm}(k)=J_{x y, \mu} \cos (k)-J_{z z, \mu} \mp \tilde{h}_{\mu} .
$$

Equation (29) describes all magnon type of excitations present in the strong-plaquette expansion of the original model, via the mapping given by Eqs. (25) and (26), and the effective couplings [Eqs. (A2)-(A15)], namely, (i) right (high field) borders of $M=0 \quad(+, \mu=1 A(B))$ and $M=\frac{1}{2} \quad(+, \mu=$ $2 A(B)$ ); (ii) left (low field) boundaries of $M=\frac{1}{2} \quad(-, \mu=$ $1 A(B))$ and $M=1 \quad(-, \mu=2 A(B))$.

Let us now consider the $M=0$ plateau in the effective model, which is associated to a double-degenerate Néel state. Here, the elemental spinon excitation consists of a spin wave composed by a linear combination of domain-wall states with two consecutive spin inversions, carrying $\Delta S_{z}=+\left(-\frac{1}{2}\right)$. Following a similar procedure as the magnon case we obtain the spinon $(S)$ dispersion

$$
\omega_{S, \mu}^{ \pm}(k)=J_{x y, \mu} \cos (2 k)+\frac{1}{2} J_{z z, \mu} \mp \frac{1}{2} \tilde{h}_{\mu} .
$$

Equivalently to the previous case, Eq. (30) describes all spinon type of excitations, namely, (i) right (high field) borders of $M=\frac{1}{4} \quad(+, \mu=1 A(B))$ and $M=\frac{3}{4} \quad(+, \mu=2 A(B)) ;(\mathrm{ii})$ left (low field) boundaries of $M=\frac{1}{4} \quad(-, \mu=1 A(B))$ and $M=\frac{3}{4} \quad(-, \mu=2 A(B))$.

From the above magnon $\omega_{M, \mu}^{ \pm}(k)$ and spinon $\omega_{S, \mu}^{ \pm}(k)$ dispersions we can calculate the critical fields, i.e., the edges and the width of the plateaus present in the model. For this, we impose the condition of gap closure (minimum of the dispersion), which allows to determine the effective fields in terms of the effective couplings and thus the critical fields according to the original couplings of the model. Due to the number of parameters of the model, in general we obtain the critical fields numerically which is much simpler compared to the DMRG or the numerical solution of the Bethe ansatz equation approaches.

The techniques used in this work to determine the critical fields, DMRG and effective models, involve different types of approximation. DMRG suffers finite-size effects and the effective models have a perturbative origin. However, these methods are somewhat complementary and provide consistent results. Although, as we have mentioned, it is not the aim of this work to quantitatively determine the edges of each plateau in the whole parameter space of the model, it is interesting to compare the results of the different techniques in some representative cases. For this purpose, in Fig. 6 we show the critical fields determined by DMRG (full magnetization curve in blue line) and the edges of the different plateaus present by applying the gap closure condition of the corresponding magnon dispersion (red dashed lines) or spinon dispersions (orange dashed lines). As can be observed, there is a very good agreement between the different techniques, which is maintained up to intermediate couplings compared with the unity $\left(J_{0}\right)$.

As previously mentioned and as can be seen in Fig. 6(c), the plateaus at $M=\frac{1}{4}$ and $\frac{3}{4}$ are small compared to the others, so that they could be difficult to detect numerically by sweeping a fairly large parameter space as in this model using DMRG. It is therefore of particular interest to be able to estimate not only the presence, but the maximum possible size of these plateaus as well as their interdependence with frustration and dimerization. From this point of view, the effective model offers a direct way of evaluating positions and maximum widths of the plateaus. This will occur when there is no intermediate Luttinger liquid phase between plateaus, i.e., the limit of the effective Ising model where $J_{x y, \mu}=0$. In this condition, the magnon and spinon dispersion bands do not 
propagate (they are flat) and the edges of fields predicted by both merge. From Eqs. (29) and (30) we see that the effective critical fields at the Ising limit satisfy

$$
J_{z z, \mu}= \pm \tilde{h}_{\mu} \quad \text { for } \quad J_{x y, \mu}=0 .
$$

Note in passing that this condition is obtained from the effective model exact line $h_{\mathrm{L}-\mathrm{F}}$ [Eq. (27)] in the limit $\Delta \rightarrow \infty$.

By solving Eqs. (31) in terms of the original variables of the model [Eqs. (A2)-(A15)] we get the following cases for the widest plateaus $\Delta h$. For case $A\left(J_{d}<\frac{2 J}{1+J}\right)$, the Ising limit is obtained for $g_{k}=K_{d}-2 K=0$ with the same $\Delta h=K / 2$ for both $M=\frac{1}{4}$ and $\frac{3}{4}$ plateaus. On the other hand, for case $B\left(J_{d}>\frac{2 J}{1+J}\right)$, the plateau widths are not the same, although we can also obtain analytical expressions for both. In the case $M=\frac{1}{4}$ the Ising condition is obtained for both $g=J_{d}-2 J=$ 0 and $g_{k}=K_{d}-2 K=0$ although these conditions are not satisfied for $M=\frac{3}{4}$.

\section{CONCLUSIONS}

In this paper, we have studied the magnetization process in a zigzag quantum antiferromagnet in the presence of both frustration and plaquette dimerization. Due to the complexity of the unit cell, several cases are present giving a rich structure of the magnetization curve. At zero magnetic field, the ground state is exactly determined under the conditions $g=J_{d}-2 J=0$ and $g_{k}=K_{d}-2 K=0$ and corresponds to a product state of spin singlets. Around this highly frustrated line we investigate the $M=0$ plateau by using dimer series expansions, bond operator mean field theory, and numerically by means of density matrix renormalization group.

In order to analyze the magnetization process, we complement the numerical results with first-order low-energy effective models starting from the limit of decoupled plaquettes. From a qualitative point of view, the effective models capture the essential features obtained numerically and bring a simple physical interpretation of the emergent structure of plateaus in the original zigzag model. In addition, the analysis of magnon and spinon excitations in the effective models allows us to obtain estimations of the critical fields bordering the plateaus, which are in a very good quantitative agrement with the numerical computations in a strong plaquette dimerization regime.

Our study also suggests that the combined effect of onplaquette frustration, controlled by $g$ and $g_{k}$ and interplaquette dimerization, via $\delta=K / J$ and $\delta_{d}=K_{d} / J_{d}$, plays a central role in the structure of the magnetization curve. In fact, whenever possible, according to the values of the couplings involved, $M=\frac{1}{4}$ and $\frac{3}{4}$ plateaus are widest along the line of maximum frustration. In this sense, we say that these plateaus are induced by frustration, which together with the dimerization between plaquettes provide a rich interplay between both aspects that are intrinsic to the model. For this reason, the maximally frustrated $g$ and $g_{k}=0$ line is not only fundamental to zero field, but plays a central role beyond zero magnetic field, which is reflected in its influence on the resulting magnetization process.

\section{ACKNOWLEDGMENTS}

We acknowledge useful discussions with M. Matera. C. A. Lamas is supported by CONICET (Grant No. PIP 1691) and ANPCyT (Grant No. PICT 2013-0009)

\section{APPENDIX A: EFFECTIVE HAMILTONIANS}

As we discuss in the main body of the text, we construct an effective Hamiltonian via degenerate perturbation theory. The energy corresponding to the states on a square plaquette is presented in Table I. By applying a standard degenerate perturbation theory around the points $h c_{\mu}$ where the lowest eigenvalues become degenerate we obtain, up to a constant term, a first-order effective Hamiltonian in the form

$$
\mathbf{H}_{\mathrm{eff}, \mu}^{(1)}=\sum_{n} J_{x y, \mu}\left(\mathbf{S}_{n}^{x} \cdot \mathbf{S}_{n+1}^{x}+\mathbf{S}_{n}^{y} \cdot \mathbf{S}_{n+1}^{y}\right)+J_{z z, \mu} \mathbf{S}_{n}^{z} \cdot \mathbf{S}_{n+1}^{z}-\tilde{h}_{\mu} \mathbf{S}_{n}^{z},
$$

where the couplings are given by

$$
\begin{gathered}
J_{x y, 1 A}=-\frac{\left(\sqrt{4 J^{2}-2 J\left(J_{d}+2\right)+J_{d}^{2}-2 J_{d}+4}+J-J_{d}+1\right)\left(2 K-K_{d}\right)}{6 \sqrt{4 J^{2}-2 J\left(J_{d}+2\right)+J_{d}^{2}-2 J_{d}+4}}, \\
J_{z z, 1 A}=\frac{1}{16}\left(2 K+K_{d}\right), \\
\tilde{h}_{1 A}=h+\frac{1}{2}\left(-\sqrt{4 J^{2}-2 J J_{d}-4 J+J_{d}^{2}-2 J_{d}+4}-J_{d}\right)+\frac{1}{16}\left(2 K+K_{d}\right), \\
J_{x y, 1 B}=\frac{K_{d}\left[\gamma(1-2 \rho)-3 J^{2}+J\left(\gamma+2 J_{d}+6\right)-2 J_{d}^{2}-J_{d}(\gamma+\rho-2)\right]+2(J-1) K\left(-3 \gamma+J-J_{d}-2 \rho+1\right)-3 K_{d}}{12 \gamma \rho}, \\
(\mathrm{A} 3) \\
J_{z z, 1 B}=\frac{1}{16}\left(\frac{J_{d}^{2}\left(K_{d}-2 K\right)}{(J-1)^{2}+J_{d}^{2}}+\frac{2 J_{d} K_{d}}{\sqrt{(J-1)^{2}+J_{d}^{2}}}+2 K+K_{d}\right),
\end{gathered}
$$




$$
\begin{gathered}
\tilde{h}_{1 B}=h+\frac{1}{2}\left(\sqrt{J^{2}-2 J+J_{d}^{2}+1}-\sqrt{4 J^{2}-2 J J_{d}-4 J+J_{d}^{2}-2 J_{d}+4}-J-1\right) \\
+\frac{1}{16}\left(-\frac{J_{d}^{2}\left(K_{d}-2 K\right)}{(J-1)^{2}+J_{d}^{2}}-\frac{2 J_{d} K_{d}}{\sqrt{(J-1)^{2}+J_{d}^{2}}}-2 K-K_{d}\right), \\
J_{x y, 2 A}=\frac{K_{d}}{4}-\frac{K}{2}, \\
J_{z z, 2 A}=\frac{K}{8}+\frac{K_{d}}{16}, \\
\tilde{h}_{2 A}=h-J-2\left(-\frac{3 K}{16}-\frac{3 K_{d}}{32}\right)-1, \\
J_{x y, 2 B}=\frac{K_{d}\left(J_{d}-\sqrt{(J-1)^{2}+J_{d}^{2}}\right)-2(J-1) K}{4 \sqrt{(J-1)^{2}+J_{d}^{2}}}, \\
J_{z z, 2 B}=\frac{1}{16}\left(\frac{J_{d}^{2}\left(K_{d}-2 K\right)}{(J-1)^{2}+J_{d}^{2}}-\frac{2 J_{d} K_{d}}{\sqrt{(J-1)^{2}+J_{d}^{2}}}+2 K+K_{d}\right), \\
\tilde{h}_{2 B}=h+\frac{1}{2}\left(-\sqrt{J^{2}-2 J+J_{d}^{2}+1}-J-J_{d}-1\right)+\frac{1}{16}\left(\frac{J_{d}^{2}\left(K_{d}-2 K\right)}{(J-1)^{2}+J_{d}^{2}}+\frac{2 J_{d} K_{d}}{\sqrt{(J-1)^{2}+J_{d}^{2}}}-3\left(2 K+K_{d}\right)\right),
\end{gathered}
$$

where

$$
\begin{gathered}
\gamma=\sqrt{(J-1)^{2}+J_{d}^{2}}, \\
\rho=\sqrt{4 J^{2}-2 J\left(J_{d}+2\right)+\left(J_{d}-2\right) J_{d}+4} .
\end{gathered}
$$

\section{APPENDIX B: DIMER SERIES EXPANSION}

In this appendix, we display the explicit expressions for ground-state energy and triplet dispersion employed in the work, for the case of homogeneous plaquettes, i.e., $\delta=\delta_{d}=1$. The method employed is the continuous unitary transformation (CUT) [29]. Here, for brevity we present explicit results to $O(6)$ although the calculations shown have been made using $O(10)$. The ground-state energy (per dimer) reads as

$$
\begin{aligned}
\operatorname{egs}= & -\frac{3}{4}-\frac{3 g^{2}}{32}-\frac{3 g^{2} J}{32}-\frac{3 g^{3}}{128}-\frac{g^{2} J^{2}}{8}-\frac{5 g^{3} J}{64}-\frac{13 g^{4}}{2048}-\frac{17 g^{2} J^{3}}{96}-\frac{73 g^{3} J^{2}}{384} \\
& -\frac{89 g^{5}}{24576}-\frac{289 g^{4} J}{6144}-\frac{155 g^{2} J^{4}}{576}-\frac{47 g^{4} J^{2}}{256}-\frac{2297 g^{5} J}{73728}-\frac{463 g^{6}}{196608},
\end{aligned}
$$

where $g=J_{d}-2 J$. Note that for $g=0$ only the first term $-\frac{3}{4}$ survives, corresponding to the dimer-product exact ground state analyzed in Sec. I. Similarly the triplet dispersion is given by $\omega_{\mathrm{SE}}=\sum_{n=0}^{\infty} c_{n} \cos (n k)$, where $c_{n}$ coefficients up to $O(6)$ are

$$
\begin{aligned}
c_{0}= & 1-\frac{g^{2}}{16}+\frac{3 g^{3}}{64}+\frac{23 g^{4}}{1024}-\frac{3 g^{5}}{256}+\frac{1273 g^{6}}{221184}-g J-\frac{g^{2} J}{16}+\frac{23 g^{3} J}{64}-\frac{43 g^{4} J}{512}-\frac{71 g^{5} J}{1536}-J^{2}-g J^{2}+\frac{55 g^{2} J^{2}}{64}+\frac{57 g^{3} J^{2}}{256} \\
& -\frac{55121 g^{4} J^{2}}{55296}-J^{3}+\frac{g J^{3}}{2}+\frac{105 g^{2} J^{3}}{64}-\frac{9239 g^{3} J^{3}}{2304}-\frac{J^{4}}{8}+\frac{99 g J^{4}}{32}-\frac{37399 g^{2} J^{4}}{6912}+\frac{7 J^{5}}{4}-\frac{361 g J^{5}}{576}+\frac{367 J^{6}}{192}, \\
c_{1}= & -\frac{g}{\mathbf{2}}-\frac{g^{2}}{4}+\frac{g^{3}}{32}+\frac{5 g^{4}}{256}-\frac{35 g^{5}}{2048}+\frac{3121 g^{6}}{221184}-g J-\frac{g^{2} J}{4}+\frac{19 g^{3} J}{64}-\frac{337 g^{4} J}{1536}-\frac{227 g^{5} J}{13824}-J^{2}-g J^{2}+\frac{15 g^{2} J^{2}}{16}-\frac{45 g^{3} J^{2}}{128} \\
& -\frac{92525 g^{4} J^{2}}{55296}-J^{3}+\frac{9 g J^{3}}{8}+\frac{25 g^{2} J^{3}}{16}-\frac{2863 g^{3} J^{3}}{384}+\frac{J^{4}}{4}+\frac{881 g J^{4}}{192}-\frac{11815 g^{2} J^{4}}{1152}+\frac{23 J^{5}}{8}-\frac{1165 g J^{5}}{576}+\frac{133 J^{6}}{48}, \\
c_{2}= & -\frac{\boldsymbol{g}^{2}}{\mathbf{1 6}}-\frac{g^{3}}{32}-\frac{15 g^{4}}{512}-\frac{283 g^{5}}{18432}+\frac{79 g^{6}}{16384}-\frac{g^{2} J}{8}-\frac{5 g^{3} J}{32}-\frac{1405 g^{4} J}{4608}-\frac{1679 g^{5} J}{36864}+\frac{g^{2} J^{2}}{64}-\frac{1243 g^{3} J^{2}}{1152}-\frac{47249 g^{4} J^{2}}{36864} \\
& +\frac{5 g J^{3}}{8}-\frac{19 g^{2} J^{3}}{32}-\frac{11933 g^{3} J^{3}}{2304}+\frac{3 J^{4}}{8}+\frac{67 g J^{4}}{48}-\frac{5419 g^{2} J^{4}}{768}+\frac{9 J^{5}}{8}-\frac{247 g J^{5}}{96}+\frac{107 J^{6}}{192},
\end{aligned}
$$




$$
\begin{aligned}
c_{3}= & -\frac{\boldsymbol{g}^{\mathbf{3}}}{\mathbf{6 4}}-\frac{g^{4}}{48}-\frac{9 g^{5}}{1024}-\frac{337 g^{6}}{147456}-\frac{g^{3} J}{12}-\frac{203 g^{4} J}{1536}-\frac{1355 g^{5} J}{36864}-\frac{5 g^{2} J^{2}}{96}-\frac{71 g^{3} J^{2}}{192} \\
& -\frac{2099 g^{4} J^{2}}{6144}-\frac{17 g^{2} J^{3}}{48}-\frac{5137 g^{3} J^{3}}{4608}-\frac{19 g J^{4}}{192}-\frac{1973 g^{2} J^{4}}{1152}-\frac{113 g J^{5}}{96}-\frac{29 J^{6}}{96}, \\
c_{4}= & -\frac{\mathbf{5 g}^{\mathbf{4}}}{\mathbf{1 0 2 4}}-\frac{67 g^{5}}{9216}-\frac{13373 g^{6}}{1769472}-\frac{67 g^{4} J}{2304}-\frac{1405 g^{5} J}{24576}-\frac{5 g^{3} J^{2}}{288}-\frac{3643 g^{4} J^{2}}{36864}-\frac{431 g^{3} J^{3}}{13824}+\frac{11 g^{2} J^{4}}{768}, \\
c_{5}= & -\frac{\mathbf{7 g}^{\mathbf{5}}}{\mathbf{4 0 9 6}}-\frac{767 g^{6}}{221184}-\frac{767 g^{5} J}{55296}-\frac{497 g^{4} J^{2}}{55296}, \\
c_{6}= & -\frac{\mathbf{2 1 g ^ { 6 }}}{\mathbf{3 2 7 6 8}} .
\end{aligned}
$$

It is interesting to compare previous SE coefficients with the BO-HP expansion $\omega_{\mathrm{HP}}=\sqrt{1-g \cos k}=\sum_{n=0}^{\infty} \tilde{c_{n}} \cos (n k)$, which to $O(6)$ reads as

$$
\begin{aligned}
& \tilde{c_{0}}=\mathbf{1}-\frac{g^{2}}{16}-\frac{15 g^{4}}{1024}-\frac{105 g^{6}}{16384}, \\
& \tilde{c_{1}}=-\frac{\boldsymbol{g}}{\mathbf{2}}-\frac{3 g^{3}}{64}-\frac{35 g^{5}}{2048}, \\
& \tilde{c_{2}}=-\frac{\boldsymbol{g}^{\mathbf{2}}}{\mathbf{1 6}}-\frac{5 g^{4}}{256}-\frac{315 g^{6}}{32768}, \\
& \tilde{c_{3}}=-\frac{\boldsymbol{g}^{\mathbf{3}}}{\mathbf{6 4}}-\frac{35 g^{5}}{4096}, \quad \tilde{c_{4}}=-\frac{\mathbf{5 \boldsymbol { g } ^ { 4 }}}{\mathbf{1 0 2 4}}-\frac{63 g^{6}}{16384}, \\
& \tilde{c_{5}}=-\frac{\mathbf{7 g}}{\mathbf{4 0 9 6}}, \quad \tilde{c_{6}}=-\frac{\mathbf{2 1 \boldsymbol { g } ^ { \mathbf { 6 } }}}{\mathbf{3 2 7 6 8}} .
\end{aligned}
$$

From Eqs. (B2) and (B3), we note that the bond operator technique is only exact at leading order (bold highlighted terms). This connection between both methods illustrates the effect of neglecting interactions beyond the quadratic order in the bosonic model [30].

[1] E. Dagotto and T. M. Rice, Science 271, 618 (1996).

[2] A. Honecker, F. Mila, and B. Normand, Phys. Rev. B 94, 094402 (2016).

[3] A. Honecker, S. Wessel, R. Kerkdyk, T. Pruschke, F. Mila, and B. Normand, Phys. Rev. B 93, 054408 (2016).

[4] N. Chepiga, F. Michaud, and F. Mila, Phys. Rev. B 88, 184418 (2013).

[5] A. Lavarélo, G. Roux, and N. Laflorencie, Phys. Rev. B 84, 144407 (2011).

[6] K. Totsuka, Phys. Rev. B 57, 3454 (1998).

[7] W. Shiramura, K.-i. Takatsu, B. Kurniawan, H. Tanaka, H. Uekusa, Y. Ohashi, K. Takizawa, H. Mitamura, and T. Goto, J. Phys. Soc. Jpn. 67, 1548 (1998).

[8] M. Matsumoto, J. Phys. Soc. Jpn. 84, 034701 (2015).

[9] D. C. Cabra, A. De Martino, A. Honecker, P. Pujol, and P. Simon, Phys. Rev. B 63, 094406 (2001).

[10] J.-B. Fouet, F. Mila, D. Clarke, H. Youk, O. Tchernyshyov, P. Fendley, and R. M. Noack, Phys. Rev. B 73, 214405 (2006).

[11] K. Okamoto, Prog. Theor. Phys. Suppl. 145, 113 (2002).

[12] J.-H. Mikeska and A. K. Kolezhuk, One Dimensional Magnetism, Lecture Notes in Physics, Vol. 645 (Springer, Berlin, 2004), Chap. 1, pp. 1-83.

[13] M. Takigawa and F. Mila, Magnetization Plateaus, Springer Series in Solid-State Sciences, Vol. 164 (Springer, Berlin, 2011), Chap. 10, pp. 241-267.
[14] M. Oshikawa, M. Yamanaka, and I. Affleck, Phys. Rev. Lett. 78, 1984 (1997).

[15] C. A. Lamas, S. Capponi, and P. Pujol, Phys. Rev. B 84, 115125 (2011).

[16] K. Totsuka, Phys. Lett. A 228, 103 (1997).

[17] A. Tanaka, K. Totsuka, and X. Hu, Phys. Rev. B 79, 064412 (2009).

[18] S. Takayoshi, K. Totsuka, and A. Tanaka, Phys. Rev. B 91, 155136 (2015).

[19] H. Hu, C. Cheng, Z. Xu, H.-G. Luo, and S. Chen, Phys. Rev. B 90, 035150 (2014).

[20] X. Plat, F. Alet, S. Capponi, and K. Totsuka, Phys. Rev. B 92, 174402 (2015).

[21] V. O. Garlea, A. Zheludev, K. Habicht, M. Meissner, B. Grenier, L.-P. Regnault, and E. Ressouche, Phys. Rev. B 79, 060404 (2009).

[22] A. Zheludev, V. O. Garlea, L.-P. Regnault, H. Manaka, A. Tsvelik, and J.-H. Chung, Phys. Rev. Lett. 100, 157204 (2008).

[23] M. Fujisawa, J.-Y. Yamaura, H. Tanaka, H. Kageyama, Y. Narumi, and K. Kindo, J. Phys. Soc. Jpn. 72, 694 (2003).

[24] W. Brenig and K. W. Becker, Phys. Rev. B 64, 214413 (2001).

[25] J. M. Matera and C. A. Lamas, J. Phys.: Condens. Matter 26, 326004 (2014).

[26] C. A. Lamas and J. M. Matera, Phys. Rev. B 92, 115111 (2015).

[27] S. Sachdev and R. N. Bhatt, Phys. Rev. B 41, 9323 (1990). 
[28] B. Bauer, L. D. Carr, H. G. Evertz, A. Feiguin, J. Freire, S. Fuchs, L. Gamper, J. Gukelberger, E. Gull, S. Guertler, A. Hehn, R. Igarashi, S. V. Isakov, D. Koop, P. N. Ma, P. Mates, H. Matsuo, O. Parcollet, G. Pawowski, J. D. Picon, L. Pollet, E. Santos, V. W. Scarola, U. Schollwck, C. Silva, B. Surer, S. Todo, S. Trebst, M. Troyer, M. L. Wall, P. Werner, and S. Wessel, J. Stat. Mech.: Theor. Exp. (2011) P05001.

[29] C. Knetter and G. Uhrig, Eur. Phys. J. B 13, 209 (2000).

[30] M. Müller and H.-J. Mikeska, J. Phys.: Condens. Matter 12, 7633 (2000).

[31] B. S. Shastry and B. Sutherland, Phys. Rev. Lett. 47, 964 (1981).

[32] T. Tonegawa and I. Harada, J. Phys. Soc. Jpn. 56, 2153 (1987).

[33] R. Chitra, S. Pati, H. R. Krishnamurthy, D. Sen, and S. Ramasesha, Phys. Rev. B 52, 6581 (1995).

[34] S. R. White, Phys. Rev. B 53, 52 (1996).

[35] S. Brehmer, H.-J. Mikeska, and U. Neugebauer, J. Phys.: Condens. Matter 8, 7161 (1996).

[36] S. Watanabe and H. Yokoyama, J. Phys. Soc. Jpn. 68, 2073 (1999).

[37] K. Okamoto and K. Nomura, Phys. Lett. A 169, 433 (1992).
[38] T. Morita and T. Horiguchi, Phys. Lett. A 38, 223 (1972).

[39] K. Okunishi and T. Tonegawa, Phys. Rev. B 68, 224422 (2003).

[40] K. Okunishi and T. Tonegawa, J. Phys. Soc. Jpn. 72, 479 (2003).

[41] A. Honecker, J. Schulenburg, and J. Richter, J. Phys.: Condens. Matter 16, S749 (2004).

[42] T. Tonegawa, K. Okamoto, K. Okunishi, K. Nomura, and M. Kaburagi, Phys. B (Amsterdam) 346, 50 (2004).

[43] T. Tonegawa, T. Nishida, and M. Kaburagi, Phys. B (Amsterdam) 246, 368 (1998).

[44] F. Mila, Eur. Phys. J. B 6, 201 (1998).

[45] K. Tandon, S. Lal, S. K. Pati, S. Ramasesha, and D. Sen, Phys. Rev. B 59, 396 (1999).

[46] D. C. Cabra and M. D. Grynberg, Phys. Rev. B 62, 337 (2000).

[47] F. Michaud, T. Coletta, S. R. Manmana, J.-D. Picon, and F. Mila, Phys. Rev. B 81, 014407 (2010).

[48] F. A. Gómez Albarracín, M. Arlego, and H. D. Rosales, Phys. Rev. B 90, 174403 (2014).

[49] H. Bethe, Z. Phys. 71, 205 (1931).

[50] J. D. Cloizeaux and M. Gaudin, J. Math. Phys. 7, 1384 (1966). 